Article

\title{
A Critical Analysis of Behavioural Crowd Dynamics-From a Modelling Strategy to Kinetic Theory Methods
}

\author{
Ahmed Elaiw ${ }^{1, *,+}+$, Yusuf Al-Turki ${ }^{2,+}$ and Mohamed Alghamdi ${ }^{1,+}$ \\ 1 Department of Mathematics, Faculty of Science, King Abdulaziz University, P.O. Box 80203, Jeddah 21589, \\ Saudi Arabia \\ 2 Department of Electrical and Computer Engineering, King Abdulaziz University, Jeddah 21589, \\ Saudi Arabia \\ * Correspondence: a_m_elaiw@yahoo.com; Tel.: +966-567573631 \\ + These authors contributed equally to this work.
}

Received: 1 May 2019; Accepted: 28 June 2019; Published: 1 June 2019

\begin{abstract}
This paper proposes a critical analysis of the literature addressed to modelling and simulations of human crowds with the aim of selecting the most appropriate scale out of the microscopic (individual based), mesoscopic (kinetic), and macroscopic (hydrodynamical) approaches. The selection is made focusing on possible applications of the model. In particular, model validation and safety problems, where validation consists of studying the ability of models to depict empirical data and observed emerging behaviors. The contents of the paper look forward to computational applications related to the flow crowds on the Jamarat bridge.
\end{abstract}

Keywords: crowd dynamics; scaling; kinetic models; safety

\section{Introduction, Motivations, and Plan of the Paper}

Our paper proposes a critical analysis of the existing literature on crowd modeling in order to select the most appropriate scale out of the microscopic (individual based), mesoscopic (kinetic), and macroscopic (hydrodynamical) scales, henceforth called micro-scale, meso-scale, and macro-scale. The selection is made focusing on possible applications of the model. Indeed, we have in mind safety problems for crowd dynamics in complex venues accounting for stress conditions and overcrowding. A strategic objective is the derivation of mathematical models suitable to depict the complexity features of the crowd and to reproduce, as far as it is possible, empirical data. Simulations are required reproduce real flow conditions and can allow to support crisis managers to improve safety conditions.

Generally, the modeling approach selects a specific scale without a preliminary study of the most appropriate one. On the other hand, the said selection is necessary to develop models appropriate to the case study under consideration. The main conceptual difficulty is induced, as mentioned in [1], by the fact that the physics, needed by the modelling and simulation of complex (living) systems, is not fully understood and mathematical models of crowd dynamics do not yet seem able to depict the whole variety of their emerging behaviors. This lack of knowledge amounts to a limited production of empirical data and makes the said selection of the scale a necessary step preliminary to the modeling approach.

The need for developing a research activity in the field of crowd dynamics is strongly motivated by the literature published in interdisciplinary journals. As an example, the review paper [2] is focused on the support to decision making towards safety of human crowds in crisis situations, see also [3,4]. Safety requires that local density remains below a critical level, which depends on the shape and the physical qualities of the venue in which the pedestrians move. More generally, crisis situations impose 
to consider high stress conditions, which can be generated by incidents $[5,6]$, as well as the role of the quality of venue on crowd movement $[7,8]$.

The presentation is mainly based on concepts which provide a general framework to support well-defined applications. Specifically, we look forward to computational applications related to the flow of crowds and safety problems on the Jamarat bridge to be treated in a forthcoming paper. In view of this application, formalizations are quite limited in this present paper. This objective requires accounting for the emotional state of the crowd, which can be heterogeneously distributed and which can have an important influence on the mechanics of the motion. The contents are as follows:

Section 2 firstly defines the main objectives of the modelling approach and of the related computational tools referred, as mentioned, to well-defined objectives. Secondly, a general modelling strategy is proposed, which is valid at all scales. Lastly, the complexity of human crowds, to be taken into account in the actual description of the collective behaviour, are reported.

Section 3 defines the general mathematical structures which are in charge of supporting the modelling approach according to the strategy defined in Section 2, where the rationale is in various steps, firstly a general structure is defined suitable for capturing the main features of the crowd and of the venue in which the crowd moves, subsequently specific models are derived inserting into the said structures specific models of the material behaviors of the crowd, and lastly a validation of models can be developed by appropriate comparisons with empirical data.

Section 4 proposes a forward look at applications, where the main objective, to be treated in a forthcoming paper [9], is the development of simulations in a venue with features analogous to those of the Jamarat bridge in Makkah.

This paper does not claim to be a survey of the existing literature which would be, however a useful general objective, but a critical analysis of a well-defined selection of articles useful to the aforementioned selection of the modelling scale. The present state of the art does not provide an exhaustive survey of crowd modeling at each scale to be followed by a critical comparisons of the achievements at each scale. Nevertheless, some surveys are available focusing separately on each scale, for instance the book [10] for the modeling at the macroscopic scale and the recent survey [11] for the modeling at the mesocopic scale. An additional bibliography is reported in Section 3.

\section{Modelling Requirements and Strategy}

Human crowds of very many individuals, as it is known, occur in venues characterized by several interconnected areas, each of them presenting different geometrical and qualitative features. An important characterization of the geometry is the presence of walls and obstacles, but also narrow corridors linking different areas, sharp changes in the path directions, and many others. We do share some definitions and specific features to characterize human crowds as they have been proposed in [12]. In the following, firstly three key questions are selected; secondly an answer, somehow inspired by [12], is given for each of them; lastly some reasonings are proposed, in italics, to enlighten a deeper vision of the problem.

- What is a crowd? Agglomeration, in the same venue, of many walkers whose collective dynamics are determined by interactions with other individuals. The assessment of a critical threshold, somewhat analogous to the Knudsen number, suitable to separate individual motion from collectively driven motion appears to be an open problem.

- Which is the most unsafe output of emotional states? The term "panic", which is occasionally criticized by experts in the field, can induce a breakdown of ordered, cooperative behaviour. Alternatively, we use the term "stress" and observe that, in many cases, stress conditions are induced by a perceived struggle for survival which drives walkers towards not safe situations rather than their survival. The concept of panic/stress should be related, as we shall see in the item below, to the concept of collective rationality/irrationality. 
- How we can define a collective intelligence? It is a collective strategy which induces an overall collective emergent behavior in a large population of walkers due to nonlinearly additive interactions which modify individual strategies and might lead to a commonly shared consensus. Consensus towards a common strategy does not imply rationality towards safe conditions, on the contrary crisis situations observe consensus towards irrational behaviors.

The answers to the three key questions will pervade our paper in the following sections consistently with the phenomenological interpretation of crowd dynamics which is delivered by the aforementioned answers and subsequent reasonings.

After these introductory reasonings, the rationale of a possible approach to modelling can be defined independently from the specific scale which is adopted towards the derivation of mathematical tools. A concise description of each step is reported below followed by the rationale and related targets, indicated in italic characters, for each step.

1. Mathematical structures to support the modelling approach: The modelling approach refers, as we shall see in the next section, to a strategic selection of one of the three scales, i.e., micro-scale (individual based), meso-scale (kinetic), and macro-scale (hydrodynamic). The selection of the scale should be developed by a preliminary analysis of the reproduction by models of the specific features of living systems. Further modelling requirements concern the validity of models and of related computational tools.

2. Derivation of a mathematical structure and modelling: All requirements reported in Item 1 should be included in a general differentials structure specialized for each scale. These structures provides the conceptual basis for the derivation of models based on a detailed analysis and modelling of walkers (pedestrians) among themselves and with walls and obstacle. In addition, the quality and main physical features of the venue, where the dynamics occur, should be taken into account.

3. Validation: Validation should be addressed to verify how far models can reproduce, quantitatively, empirical data and, qualitatively, expected emerging behaviors. The former specifically refers to the velocity diagram, representing mean velocity versus density. The latter is based on the empirical observation that collective motions exhibit a self-organization ability leading to patterns which are reproduced qualitatively, but it might be subject to large quantitative deviations for small variations of the flow conditions.

4. Computing: After validation, computational codes should be developed. As it is known [13], different mathematical structures, hence different computational tools, correspond to each scale.

5. Simulations to support crisis managers: Managers can use simulations with various specific purposes, namely "training", to support decision making. In addition, simulations can be used to improve the design of venues by comparing venues which, with equal transport ability, induce situations of minor overcrowding.

Let us now define the complexity features of crowds which have been object in [14] of sharp reasonings:

- Strategy: Individual walkers can develop strategies which take into account the geometry of the venue and the interactions with the surrounding walkers.

- Heterogeneity: The ability to express a strategy is not the same for all walkers. This strategy includes different walking targets and rules in the crowd, for instance, in evacuation it includes the possible presence of leaders.

- Learning ability: Living systems receive inputs from their environments and have the ability to learn from past experience. Therefore their strategic ability and the rules of interactions can evolve in time. Stress conditions can induce important modifications to collective behaviors.

- Nonlinear Interactions: Interactions are nonlinearly additive and involve neighbors in the sensitivity zone of each walker. In some cases, also distant walkers manage to communicate. Walkers perceive, at distance, walls and obstacles and modify their walking strategy accordingly. 
- Quality of the venue: The walking strategy, and hence the overall dynamics, is affected by the quality of the venue, where they move, for instance, environment, weather conditions, geometry, and specific features of the venue.

All aforementioned features should be taken into account, as far as it is possible, in the selection of the representation and modelling scale and subsequently in the derivation of mathematical and computational tools.

It is worth stressing that the aforementioned selection should also be related to the specific applications which are planned. Considering that the forthcoming objective is the simulation of the dynamics in the Jamarat bridge bearing in mind possible incidents, the level of emotional state is an important feature to be accounted for. In addition, heterogeneity is also important as the propagation of emotional states is extremely rapid and can, consequently, lead to disasters.

\section{On the Selection of the Mesoscopic (Kinetic) Scale}

This section deals with the selection of the kinetic theory approach out of the three classical representation and modelling scales:

- Micro-scale (individual based) corresponding to a system with finite number of degrees of freedom, where pedestrians are individually identified by their position and velocity, while rotational motion is generally neglected.

- Meso-scale (kinetic), where the micro-scale state is identified, as in individual based models, by position and velocity, but the dependent variable the probability distribution function over the micro-scale state.

- Macro-scale (hydrodynamic), where the dependent variable is defined by locally averages quantities, typically density and momentum.

The selection of the modelling scale is based on a detailed analysis on the ability of each structure to capture the complexity features of human crowds. The presentation is proposed in the following sequential steps treated in the following subsections:

(i) Assessment of the mathematical structures;

(ii) Rationale towards the validation of models;

(iii) Overview of the existing literature;

(iv) Selection of the kinetic theory approach;

(v) Critical analysis.

\subsection{Mathematical Structures}

Let us consider the general mathematical framework corresponding to the aforementioned modelling scale. This presentation mainly refer to [1], where a multiscale vision of crowd modelling is proposed. We consider a system of $N$ walkers and report, for each scale, the structures involving only the independent and dependent variables, while parameters are not here indicated as these appears in specific models. Our focus is on second order models which refer to accelerations.

Micro-scale: The state of the system is given by position $\mathbf{x}_{i}=\mathbf{x}_{i}(t)=\left(x_{i}(t), y_{i}(t)\right)$ and velocity $\mathbf{v}_{i}=\mathbf{v}_{i}(t)=\left(v_{x}^{i}(t), v_{y}^{i}(t)\right)$ of all $i$-th walkers with $i=1, \ldots, N$. The derivation of models requires the specific modellling the acceleration $\mathbf{F}_{i}(\cdot)$ impressed to each walker by the action of the other interacting walkers. The equation modelling this dynamics is as follows:

$$
\frac{d \mathbf{v}_{i}}{d t}=\mathbf{F}_{i}(\mathbf{x}, \mathbf{v}),
$$


where $\mathbf{x}=\left\{\mathbf{x}_{1}, \ldots, \mathbf{x}_{N}\right\}$, and $\mathbf{v}=\left\{\mathbf{v}_{1}, \ldots, \mathbf{v}_{N}\right\}$, while position and velocity are related, under regularity assumptions, by $\frac{d \mathbf{x}_{i}}{d t}=\mathbf{v}_{i}$. In particular the psycho-mechanical acceleration $\mathbf{F}_{i}(\cdot)$ depends on the overall geometry of the venue, as walkers visualize the walls and attempt not to collide with them.

Macro-scale: The state of the is represented by the local density $\rho=\rho(t, \mathbf{x})$ and the mean velocity $\xi=\xi(t, \mathbf{x})$. The derivation of models requires the specific modelling the acceleration $\mathcal{A}(\cdot)$ impressed the walkers in the elementary volume $\mathbf{x}$ the action the surrounding walkers. The equation modelling this dynamics is a follows:

$$
\partial_{t} \xi+\left(\xi \cdot \nabla_{\mathbf{x}}\right) \xi=\mathcal{A}(\rho, \xi),
$$

where the relation between density and mean velocity is defined by the equation of mass conservation:

$$
\partial_{t} \rho+\nabla_{\mathbf{x}} \cdot(\rho \xi)=0
$$

Also in this case $\mathcal{A}$ depends on the geometry of the venue as walkers, as above, visualize the walls and attempt not to collide with them.

Meso-scale: The state of system is delivered by a statistical distribution function over the micro-scale state of walkers, namely their position $\mathbf{x}$ and velocity $\mathbf{v}$ which are the micro-scale variable. As in the classical kinetic theory, macroscopic quantities are obtained by velocity weighted moments, see [15].

The mathematical structure deemed to provide the dynamics is obtained by a balance of micro-scale entities in the elementary volume of the space of micro-scale state, namely by equating the transport of $f$ to the net flow in the said micro-volume as induced by interactions:

$$
\left(\partial_{t}+\mathbf{v} \cdot \nabla_{\mathbf{x}}+\mathbf{E}(t, \mathbf{x}) \nabla_{\mathbf{v}}\right) f(t, \mathbf{x}, \mathbf{v})=(Q)(f, f)(t, \mathbf{x}, \mathbf{v}),
$$

where $\mathbf{E}$ is an external field made visual and vocal signals, while $Q$ models interactions which are nonlinear and nonlocal.

\subsection{Rationale towards Validation}

This subsection expands the brief concepts on model validation introduced in Section 2 referring to the rationale developed in [16] with focus on the kinetic theory approach. Let us firstly observe that the modelling criteria are precisely the same at all scales and specifically consists in the fulfillment of the three main abilities:

1. Capturing the complexity features of human crowds.

2. Depicting, as an emerging behavior, the velocity diagrams of crowd traffic depending on environmental conditions can determine different observable dynamics.

3. Reproduce "qualitatively" emerging behaviors which are observed in experiments.

The analysis of the literature has indicates that the following type of empirical data can be recovered:

- Velocity diagrams: These diagrams $[17,18]$ are influenced by the quality features of the venue, in addition empirical data present evidence of heterogeneous behaviors even in the case of uniform steady state uniform flow $[19,20]$.

- Walking behaviors: Empirical results have been proposed in [21-23], which can contribute to derive models.

- Development of technologies towards the individual identification of walkers [24,25].

In addition, some recent contributions have proposed specific techniques towards the tuning of models, namely parameter assessment, in $[26,27]$, where a conceptual difficulty consists in matching empirical data at the macro-scale the the dynamics at the micro-scale. 


\subsection{Overview of the Existing Literature}

The scientific and technical literature on crowd modelling and simulation is already vast and it is rapidly growing due to the impact that these studies can have on the wellbeing of the society. A critical analysis of this literature is here presented without claiming completeness, while a selection is made of contributions that appear important to the aims of our paper, colorblue namely the selection of the modelling scale.

Let us focus on the modelling at the micro-scale, where Equation (1) needs to be implemented by models of interactions between each individual with the others. Interactions with walls and trend towards the exit are generally modeled by simple rules. A model which has been well received by the scientific community is the so called social force model proposed by Helbing and coworkers [28], which has been later developed to model evacuation dynamics [12] and panic situations [5]. Still not exhaustively treated appears to be the modelling of heterogeneity, which is an important feature of crowd dynamics.

The modelling at the macro-scale is reviewed in the survey [29]. The modelling difficulty difficulty consists in understanding how the crowd selects the velocity direction and the speed by which pedestrians move. The approach, firstly proposed in [30], suggests to compute optimal trajectories by which pedestrians reach the exit. Detailed calculations are made in steady flow. More complex appears to be the problem of modelling irrational behaviors in panic situations. Additional results are reviewed in the book [10], which provides an exhaustive survey of the existing literature in the field mainly focused at the micro-scale and macro-scale.

The surveys $[13,31]$ introduce the concept of the crowds as a complex system, however, this challenging problem has not been yet exhaustively treated. In fact, heterogeneous behaviors are not taken into account by hydrodynamic models, where the averaging process suppress naturally heterogeneity. An additional problem consists is that the continuity assumption, which is typical of the hydrodynamic approach, is not consistent with physical reality of a multi-particle system where rarefied flows coexist with almost continuous flows. Paper [32] the two different descriptions suggests to weight by a parameter. However the actual modelling of this parameter remains an open problem. An interesting connection between individual based models and statistical description, in view of the kinetic theory approach is proposed in [33].

Let us now consider the modelling at the meso-scale, namely the kinetic theory approach which is far less developed than that of the micro-scale and macro-scale. Indeed, this approach has been only recently developed arguably due to the conceptual difficulties to tackle this approach. However, the methodological approach to model living systems proposed in the book [15] has contributed to this challenging aim.

The motivations to develop a kinetic theory approach were already posed in [13], however the first important contribution was given in [34] for a crowd in unbounded domains. Modelling interactions with walls and obstacles has been developed in [14], where the application has shown that two counter flows moving in a corridor, even if initially are randomly distributed, subsequently segregate into two main streams one on the left moving along one of the directions and one stream in the opposite direction. In addition two numerically minor streams move along the walls in directions opposite to that of the two main streams.

Validation of the model has been developed in [16] by showing that the the model has the ability to reproduce fundamental diagrams and observed collective emerging behaviors. Further analysis on this approach will be given in the following, referring to the selection of this scale for the modelling approach and to a specific model which will be presented later.

\subsection{Selection of the Modelling Scale}

Let us consider the selection of the most appropriate modelling scale. This choice plays a key role in the further interpretation of the remaining literature still to be examined. The analysis of the existing literature already leads to the selection of the scale which is more appropriate to achieve the objectives 
defined in Section 2. In details, the meso-scale has shown abilities to capture the complexity features of human crowd which have been presented in Section 3.1. In fact, the heterogeneous behavior of walkers can be taken into account by the dependent variable of the structure, namely a probability distribution, while the use of methods of stochastic game theory in the modelling of interactions allows to depict a broad variety of possible trends of walkers related to the interactions among themselves as well as with the geometrical and quality features venues. Different typologies of walkers from leaders to groups that need physical support to evacuate are taken into account by the partition into functional subsystems.

In principle, the said complexity features can also be inserted in models at the micro-scale. However, focusing on heterogeneity, the cost of including this specific feature is definitely high both in modelling and computing, while the local averaging needed by the derivation of hydrodynamical models eliminate local heterogeneity.

However, a technical difficulty is that that while the literature on vehicular traffic shows a valuable record of results which are documented in the excellent book by Kerner [35], which reports also about interesting emerging behaviors, as an example synchronization phenomena [36], the literature on emerging behaviors in crowd dynamics is comparatively quite limited. Nevertheless we can indicate some interesting recent contributions, in particular on the interaction between crowds and venues [37], the role of social information [38], and coarse-grained data analysis [39]. These papers support the selection developed in the following.

These reasonings support the selection of the kinetic theory approach to crowd modeling. In fact, the kinetic theory approach has the ability of capturing the afore mentioned complexity features of the crowds, of reproducing the so-called velocity diagram as an emerging behavior of interactions as shown in [16], accounting for emotional states and physical quality of the venues. These features have been enlightened as shown in [37-39], while even in the case of the approach at the hydrodynamical level models have to tackle the problem of micro-macro interactions [40,41], which can be taken into account by the kinetic theory approach.

However, the modeling approach should bear in mind multiscale problems referring, for instance, to [32], where the authors suggest to weight by an appropriate parameter the micro-scale and macro-scale description, as well as to [42] on the micro-macro derivation, and to the multiscale vision proposed in [1], where it is shown how the derivation of models at each scale is derived according to the same principles and using the same parameters.

\section{Towards Modelling Perspectives and Applications}

This final section looks ahead to research perspectives and applications. In more detail, the following topics are treated:

1. Computational tools;

2. Analytic problems;

3. Support to crisis managing.

These topics are treated in the next subsections focusing on the contribution that the kinetic theory approach can give to tackle them.

\subsection{Computational Tools}

As it is known, simulations related to classical models of the kinetic theory have been developed, since the pioneer paper by Bird [43] by Monte Carlo particle methods see also [44-47] and many others. Models of human crowds introduce additional difficulties related to the interactions which do not satisfy conservation rules besides preservation of the number of particles. However, these difficulties have been tackled in [14,16], where interesting simulations, for instance on the counter-flows in long corridors, have also been proposed towards model validation. 
Interactions between pedestrians consistent with the specific features of human crowds have been taken into account in the simulations developed in $[14,16]$. Further improvements have been produced in [48] to account for the propagation of stress conditions which can be modeled by complex learning collective learning processes [49] and contagion dynamics [50].

The computational cost, in terms of computing time can be reduced if the model is simplified, for instance by discrete velocity directions, classical deterministic methods can be applied, for instance splitting methods for nonlinear transport equations [51] or finite differences [34,52]. The use of discrete models both for velocity and space has been introduced in [53] in the simpler case of vehicular traffic, where space is made discrete simply by tracts. Crowd dynamics requires a discrete areas made by triangles, while transport dynamics can be computed analogously to [53]. In parallel, developments of discrete models can contribute to modeling junctions in networks $[54,55]$.

\subsection{Analytic Problems}

Mathematical models of crowd dynamics have an impact also on the development of the research activity on fundamental aspects of mathematical research. Let us briefly mention some of these developments. In fact the two problems presented in the following refer, respectively, on the solutions of mathematical problems and on the micro-macro of hydrodynamical models. In both cases, we indicate as possible research perspectives some open problems.

The initial value problem for kinetic crowd dynamic models has been studied in [34] for a model in the whole space. The main achievement has been the proof of existence and regularity in the large of the solutions to the initial value problem with physical bounds on the density. Namely, the proof must show that the density is bounded by the maximum physical value corresponding to full packing of individuals (as they have a dimension, that is, they are not points). On the other hand, the initial-boundary value problem for a crowd in domains which include walls is still waiting for a solution.

An additional problem is the derivation of hydrodynamical models by asymptotic perturbation methods developed by letting to zero the distance between walking individuals. Analytic results have been obtained in [42] still for a crowd in the whole space, while imposing boundary conditions creates conceptual difficulties which have not found yet a mathematical response even in the relatively simpler case of one dimensional flows [56]. The approach can be developed by expanding the probability distribution function in terms of a small parameter obtained by writing the model in dimensionless form and by letting to zero the distance between individuals. Then hydrodynamical equations are obtained by averaging terms with the same power of the aforementioned parameter, see $[57,58]$. A key problem consists in deriving hyperbolic models accounting for finite propagation speed, namely by transferring to crowd models the conceptual approach developed for swarms [59] and cellular dynamics.

\subsection{Safety Problems to Support to Crisis Managers}

Let us now analyze the literature focused on crisis management, namely on the support to the decision process of crisis managers when crisis conditions appear, thus creating overcrowded flows. In these situations, the support to the crisis manager is needed to select the most appropriate strategy to achieve safety.

The onsets of crisis situations are generally not predictable, however some concomitant causes can generate them. For instance, the quality and geometry of the venues where walkers move might enhance the propagation of panic conditions, as well as an excessive concentration of individuals in the same place. If simulations can provide accurate results, then the contribution to crisis managers can be effective to investigate possible actions suitable to reduce the aforementioned excessive concentrations. This topic will be specifically treated in the forthcoming paper [9] devoted to the development of simulations in a venue somehow related to that pilgrims find themselves in on the Jamarat bridge. More general simulations can contribute to the design of infrastructures [60] and structural safety [61]. 
An interesting research perspective consists of developing computational codes suitable to account for the propagation of emotional states from localized zones, where high stress conditions are generated by incidents. The kinetic theory approach has shown the ability to depict these specific dynamics as reported in the very recent paper [48]. The perspective to be pursued consists of developing computations in high density situations and complex geometry as they appear in the Jamarat bridge, which is the main objective of our research perspective.

Author Contributions: Conceptualization, A.E. and Y.A.-T.; methodology, A.E. and M.A.; formal analysis, M.A.; investigation, Y.A.-T.; writing original draft preparation, M.A.; writing review and editing, A.E.; project administration, A.E.

Funding: This work was funded by King Abdulaziz City for Science and Technology (KACST), Saudi Arabia, under grant No. (SP-36-200). The authors, therefore, acknowledge with thanks KACST for technical and financial support.

Conflicts of Interest: The authors declare no conflict of interest.

\section{References}

1. Aylaj, B.; Bellomo, N.; Gibelli, L.; Reali, A. On a unified multiscale vision of behavioral crowds. Math. Model. Methods Appl. Sci. 2019, 29, in press.

2. Wijermans, N.; Conrado, C.; van Steen, M.; Martella, C.; Li, J. A landscape of crowd management support: An integrative approach. Saf. Sci. 2016, 86, 142-164. [CrossRef]

3. Bellomo, N.; Clark, D.; Gibelli, L.; Townsend, P.; Vreugdenhil, B.J. Human behaviours in evacuation crowd dynamics: From modelling to big data toward crisis management. Phys. Life Rev. 2016, 18, 1-21. [CrossRef] [PubMed]

4. Pelechano, N.; Badler, N.I. Modeling crowd and trained leader behavior during building evacuation. IEEE Comput. Graph. Appl. 2006, 26, 80-86. [CrossRef] [PubMed]

5. Helbing, D.; Farkas, I.; Vicsek, T. Simulating dynamical feature of escape panic. Nature 2000, 407, 487-490. [CrossRef] [PubMed]

6. Lin, J.; Luckas, T.A. A particle swarm optimization model of emergency airplane evacuation with emotion. Netw. Hetherog. Media 2015, 10, 631-646. [CrossRef]

7. Ronchi, E.; Kuligowski, E.D.; Nilsson, D.; Peacock, R.D.; Reneke, P.A. Assessing the verification and validation of building fire evacuation models. Fire Technol. 2016, 52, 197-219. [CrossRef]

8. Ronchi, E.; Reneke, P.A.; Peacock, R.D. A conceptual fatigue-motivation model to represent pedestrian movement during stair evacuation. Appl. Math. Model. 2016, 40, 4380-4396. [CrossRef]

9. Elaiw, A.M.; Al-Turki, Y.; Alghamdi, M.A. Simulations by Kinetic Models of Human Crowds on Bridges With Internal Obstacles. Work in Progress.

10. Cristiani, E.; Piccoli, B.; Tosin, A. Multiscale Modeling of Pedestrian Dynamics; Springer: Milan, Italy, 2014.

11. Albi, G.; Bellomo, N.; Fermo, L.; Ha, S.-Y.; Kim, J.; Pareschi, L.; Poyato, D.; Soler, J. Traffic, crowds, and swarms. From kinetic theory and multiscale methods to applications and research perspectives. Math. Model. Methods Appl. Sci. 2019, 29. [CrossRef]

12. Helbing, D.; Johansson, A. Pedestrian crowd and evacuation dynamics. In Enciclopedia of Complexity and System Science; Springer: Berlin, Germany, 2009; pp. 6476-6495.

13. Bellomo, N.; Dogbè, C. On the modeling of traffic and crowds: A survey of models, speculations, and perspectives. SIAM Rev. 2011, 53, 409-463. [CrossRef]

14. Bellomo, N.; Gibelli, L. Toward a behavioral-social dynamics of pedestrian crowds. Math. Model. Methods Appl. Sci. 2015, 25, 2417-2437. [CrossRef]

15. Bellomo, N.; Bellouquid, A.; Gibelli, L.; Outada, N. A Quest Towards a Mathematical Theory of Living Systems; Birkhauser-Springer: New York, NY, USA, 2017.

16. Bellomo, N.; Gibelli, L. Behavioral crowds: Modeling and Monte Carlo simulations toward validation. Comput. Fluids 2016, 141, 13-21. [CrossRef]

17. Schadschneider, A.; Seyfried, A. Empirical results for pedestrian dynamics and their implications for Cellular Automata models. In Pedestrian Behavior-Models, Data Collection and Applications; Emerald Group Publishing: Bingley, West Yorkshire, UK, 2009; pp. 27-44. 
18. Schadschneider, A.; Seyfried, A. Empirical results for pedestrian dynamics and their implications for modeling. Netw. Heterog. Media 2011, 6, 545-560.

19. Schadschneider, A.; Chraibi, M.; Seyfried, A.; Tordeux, A.; Zhang, J. Pedestrian dynamics: From empirical results to modeling. In Crowd Dynamics Voume 1-Theory Models and Safety Problems; Gibelli, L., Bellomo, N., Eds.; Springer: Berlin, Germany, 2018; pp. 63-102.

20. Seyfried, A.; Steffen, B.; Klingsch, W.; Boltes, M. The fundamental diagram of pedestrian movement revisited. J. Stat. Mech. Theory Exp. 2006, 360, 232-238. [CrossRef]

21. Daamen, W.; Hoogedorn, S.P. Experimental research of pedestrian walking behavior. In Proceedings of the TRB Annual Meeting CD-ROM, Washington, DC, USA, 22-26 January 2006.

22. Moussaid, M.; Helbing, D.; Garnier, S.; Johanson, A.; Combe, M.; Theraulaz, G. Experimental study of the behavioral underlying mechanism underlying self-organization in human crowd. Proc. R. Soc. B Biol. Sci. 2009, 276, 2755-2762. [CrossRef] [PubMed]

23. Moussaïd, M.; Theraulaz, G. Comment les piétons marchent dans la foule. La Recherche 2011, 450, 56-59.

24. Kirchner, A.; Schadschneider, A. Simulation of evacuation processes using a bionics-inspired cellular automaton model for pedestrian dynamics. Physics A 2002, 312, 260-276. [CrossRef]

25. Roggen, D.; Wirz, M.; Tröster, G.; Helbing, D. Recognition of crowd behavior from mobile sensors with pattern analysis and graph clustering methods. Netw. Heterog. Media 2011, 6, 521-544.

26. Corbetta, A.; Mountean, A.; Vafayi, K. Parameter estimation of social forces in pedestrian dynamics models via probabilistic method. Math. Biosci. Eng. 2015, 12, 337-356.

27. Corbetta, A.; Bruno, L.; Mountean, A.; Yoschi, F. High statistics measurements of pedestrian dynamics, models via probabilistic method. Transp. Res. Proc. 2014, 2, 96-104. [CrossRef]

28. Helbing, D.; Molnár, P. Social force model for pedestrian dynamics. Phys. Rev. E 1995, 51, 4282-4286. [CrossRef]

29. Hughes, R.L. The flow of human crowds. Annu. Rev. Fluid Mech. 2003, 169-182. [CrossRef]

30. Hughes, R.L. A continuum theory for the flow of pedestrians. Transp. Res. B 2002, 36, 507-536. [CrossRef]

31. Bellomo, N.; Piccoli, B.; Tosin, A. Modeling crowd dynamics from a complex system viewpoint. Math. Model. Methods Appl. Sci. 2012, 22, 1230004. [CrossRef]

32. Cristiani, E.; Piccoli, B.; Tosin, A. Multiscale modeling of granular flows with application to crowd dynamics. Multiscale Model. Simul. 2011, 9, 155-182. [CrossRef]

33. Helbing, D. Traffic and related self-driven many-particle systems. Rev. Mod. Phys. 2001, 73, $1067-1141$. [CrossRef]

34. Bellomo, N.; Bellouquid, A.; Knopoff, D. From the micro-scale to collective crowd dynamics. Multiscale Model. Simul. 2013, 11, 943-963. [CrossRef]

35. Kerner, B. The Physics of Traffic; Springer: New York, NY, USA; Heidelberg, Germany, 2004.

36. Kerner, B.; Klenov, S. A microscopic model for phase transitions in traffic flow. J. Phys. A 2002, 35, 31-43. [CrossRef]

37. Pinter-Wollman, N.; Penn, A.; Theraulaz, G.; Fiore, S.M. Interdisciplinary approaches for uncovering the impacts of architecture on collective behaviour. Philos. Trans. R. Soc. B Biol. Sci. 2018, 373, 20170232. [CrossRef]

38. Jayles, B.; Kim, H.R.; Escobedo, R.; Cezera, S.; Blanchet, A.; Kameda, T.; Sire, C.; Theraulaz, G. How social information can improve estimation accuracy in human groups. Proc. Natl. Acad. Sci. USA 2017, 114, 12620-12625. [CrossRef]

39. Motsch, S.; Moussaïd, M.; Guillot, E.G.; Moreau, M.; Pettré, J.; Theraulaz, G.; Appert-Rolland, C.; Degond, P. Modeling crowd dynamics through coarse-grained data analysis. Math. Biosci. Eng. 2018, 15, 1271-1290. [CrossRef] [PubMed]

40. Colombo, R.; Lécureux-Mercier, M. An analytical framework to describe the interactions between individuals and a continuum. J. Nonlinear Sci. 2012, 22, 39-61. [CrossRef]

41. Degond, P.; Appert-Rolland, C.; Moussaid, M.; Pettré, J.; Theraulaz, G. A hierarchy of heuristic-based models of crowd dynamics. J. Stat. Phys. 2013, 152, 1033-1068. [CrossRef]

42. Bellomo, N.; Bellouquid, A. On multiscale models of pedestrian crowds from mesoscopic to macroscopic. Commun. Math. Sci. 2015, 13, 1649-1664. [CrossRef]

43. Bird, G.A. Molecular Gas Dynamics and the Direct Simulation of Gas Flows; Oxford University Press: Oxford, UK, 1994. 
44. Aristov, V.V. Direct Methods for Solving the Boltzmann Equation and Study of Nonequilibrium Flows; Springer: New York, NY, USA, 2001.

45. Barbante, P.; Frezzotti, A.; Gibelli, L. A kinetic theory description of liquid menisci at the microscale. Kinet. Relat. Model. 2015, 8, 235-254.

46. Dimarco, G.; Pareschi, L. Numerical methods for kinetic equations. Acta Numer. 2014, 23, 369-520. [CrossRef]

47. Pareschi, L.; Toscani, G. Interacting Multiagent System. Kinetic Equations and Monte Carlo Methods; Oxford University Press: Oxford, UK, 2013.

48. Bellomo, N.; Gibelli, L.; Outada, N. On the interplay between behavioral dynamics and social interactions in human crowds. Kinet. Relat. Model. 2019, 12, 397-409. [CrossRef]

49. Burini, D.; de Lillo, S.; Gibelli, L. Stochastic differential "nonlinear" games modeling collective learning dynamics. Phys. Life Rev. 2016, 16, 123-139. [CrossRef] [PubMed]

50. Wang, L.; Short, M.; Bertozzi, A.L. Efficient numerical methods for multiscale crowd dynamics with emotional contagion. Math. Model. Methods Appl. Sci. 2017, 27, 205-230. [CrossRef]

51. Kim, D.; Quaini, A. A kinetic theory approach to model pedestrian dynamics in bounded domains with obstacles. arXiv 2019, arXiv:1901.07620v2.

52. Agnelli, J.P.; Colasuonno, F.; Knopoff, D. A kinetic theory approach to the dynamics of crowd evacuation from bounded domains. Math. Model. Methods Appl. Sci. 2015, 25, 109-129. [CrossRef]

53. Fermo, L.; Tosin, A. A fully-discrete-state kinetic theory approach to modeling vehicular traffic. SIAM J. Appl. Math. 2013, 73, 1533-1556. [CrossRef]

54. Akbarzadeh, M.; Estrada, E. Communicability geometry captures traffic flows in cities. Nat. Hum. Behav. 2018, 2, 645-652. [CrossRef]

55. Bressan, A.; Canic, S.; Garavello, M.; Herty, M.; Piccoli, B. Flows on networks: Recent results and perspectives. EMS Surv. Math. Sci. 2014, 1, 47-111. [CrossRef]

56. Bellomo, N.; Bellouquid, A.; Nieto, J.; Soler, J. On the multiscale modeling of vehicular traffic: From kinetic to hydrodynamics. Discret. Contin. Dyn. Syst. Ser. B 2014, 19, 1869-1888. [CrossRef]

57. Burini, D.; Chouhad, N. Hilbert method toward a multiscale analysis from kinetic to macroscopic models for active particles. Math. Model. Methods Appl. Sci. 2017, 27, 1327-1353. [CrossRef]

58. Burini, D.; Chouhad, N. A multiscale view of nonlinear diffusion in biology: From cells to tissues. Math. Model. Methods Appl. Sci. 2019, 29, 791-823. [CrossRef]

59. Poyato, D.; Soler, J. Euler-type equations and commutators in singular and hyperbolic limits of kinetic Cucker-Smale models. Math. Model. Methods Appl. Sci. 2017, 27, 1089-1152. [CrossRef]

60. Hoogendoorn, L.; Bovy, P.; Daamen, W. Walking infrastructure design assignment by continuous space dynamic assignment modeling. J. Adv. Transp. 2004, 38, 69-92. [CrossRef]

61. Venuti, F.; Bruno, L. Crowd structure interaction in lively footbridges under synchronous lateral excitation: A literature review. Phys. Life Rev. 2009, 6, 176-206. [CrossRef]

(C) 2019 by the authors. Licensee MDPI, Basel, Switzerland. This article is an open access article distributed under the terms and conditions of the Creative Commons Attribution (CC BY) license (http:/ / creativecommons.org/licenses/by/4.0/). 